PROCEEDINGS OF THE

AMERICAN MATHEMATICAL SOCIETY

Volume 137, Number 11, November 2009, Pages 3763-3768

S 0002-9939(09)09939-0

Article electronically published on May 27, 2009

\title{
A KATZNELSON-TZAFRIRI TYPE THEOREM IN HILBERT SPACES
}

\author{
ZOLTÁN LÉKA
}

(Communicated by Nigel J. Kalton)

\begin{abstract}
Our aim is to characterize, via an ergodic condition, the norm convergence $\lim _{n \rightarrow \infty}\left\|T^{n} Q\right\|=0$ when $T$ is a power-bounded operator on a Hilbert space and $Q$ commutes with $T$. We shall also prove that if $f \in A^{+}(\mathbb{T})$ and $Q=f(T)$, the given condition is equivalent to the vanishing of $f$ on the peripheral spectrum of $T$.
\end{abstract}

\section{INTRODUCTION}

Let us consider a complex Banach space $\mathcal{X}$ and let $T$ be a power-bounded operator on $\mathcal{X}$, i.e. $\sup _{n \geq 1}\left\|T^{n}\right\|<\infty$ holds. Then it is a simple matter to give a characterization of the norm stability of $T$ (that is, $\lim _{n \rightarrow \infty}\left\|T^{n}\right\|=0$ ) via the spectral radius formula. However, it is more interesting to give necessary and sufficient conditions for the uniform convergence

$$
\lim _{n \rightarrow \infty}\left\|T^{n} Q\right\|=0
$$

with some bounded operator $Q$, as this is far from trivial. The case $Q=T-I$, which is of great importance because of its role in the uniform zero-two law (cf. [13]) and its connection with the Gelfand-Hille theorem (see [22]), was first essentially characterized by J. Esterle [8]. This result was later found independently and generalized by Y. Katznelson and L. Tzafriri.

The celebrated Katznelson-Tzafriri theorem asserts that if $T$ is a power-bounded operator acting on a Banach space $\mathcal{X}$ and if $f(z)=\sum_{k=0}^{\infty} a_{k} z^{k}$ is a power series with absolutely convergent coefficients, which is of spectral synthesis with respect to the peripheral spectrum of $T$, then $\lim _{n \rightarrow \infty}\left\|T^{n} f(T)\right\|=0$ (see [13]). In the Hilbert space setting a richer functional calculus can be defined for contractions due to von Neumann's inequality. It was proved in 9 ] that if $f$ is an element of the disk algebra, then $f$ vanishes on the peripheral spectrum of $T$ if and only if $\lim _{n \rightarrow \infty}\left\|T^{n} f(T)\right\|=0$. On the other hand, contractions even admit an $H^{\infty}$ calculus on the unit disk. Let $T$ be a completely nonunitary contraction on a Hilbert space and let $f$ be a bounded holomorphic function on the unit disk. Then the convergence $\lim _{n \rightarrow \infty}\left\|T^{n} f(T)\right\|=0$ holds if $\lim _{r \rightarrow 1} f\left(r e^{i \theta}\right)=0$ for every $e^{i \theta}$

Received by the editors September 2, 2008, and, in revised form, February 20, 2009.

2000 Mathematics Subject Classification. Primary 47A35, 46B08; Secondary 46M07, 47B99.

Key words and phrases. Ultrapower, stablity of operators, uniform ergodicity.

This study was partially supported by Hungarian NSRF (OTKA) grant No. T 49846 and by the Marie Curie "Transfer of Knowledge" programme, project TODEQ.

(C)2009 American Mathematical Society Reverts to public domain 28 years from publication 
in the peripheral spectrum of $T$. However, an example shows that the converse implication is not true (see [4]).

Later, many extensions of the above results were proved in the discrete case as well as in the continuous one. For details, see [3, [10, 15, [17, 18, [21] and related survey articles [1], [6].

In this paper we shall prove that the assumption made in the Katznelson-Tzafriri theorem can be weakened in Hilbert spaces, and we shall provide a complete characterization of the condition $\lim _{n \rightarrow \infty}\left\|T^{n} Q\right\|=0$ whenever $Q$ commutes with $T$. We note that all former extensions of the Katznelson-Tzafriri theorem are related to bounded functional calculi of $T$ or elements of the Banach algebra generated by $T$.

The proof shall partly follow Vũ's method ([20, [21]); that is, we will first verify convergence in the strong operator topology by reducing the problem to isometries. After that we shall complete the proof using some aspects of an ultrapower approach.

\section{Preliminaries AND the MAin Result}

Let $\mathcal{L}(\mathcal{X})$ be the algebra of all bounded linear operators on a Banach space $\mathcal{X}$. Let $\sigma(T)$ stand for the spectrum of $T \in \mathcal{L}(\mathcal{X})$ and let $I$ be the identity operator on $\mathcal{X}$. Next, let $A^{+}(\mathbb{T})$ denote the set of sums of power series on the unit circle $\mathbb{T}$ whose coefficients are absolutely convergent. Then, for each power-bounded operator $T$, a bounded functional calculus naturally arises which is defined by $f(T):=\sum_{k=0}^{\infty} a_{k} T^{k} \in \mathcal{L}(\mathcal{X})$, where $f(z)=\sum_{k=0}^{\infty} a_{k} z^{k}$ and $\sum_{k=0}^{\infty}\left|a_{k}\right|<\infty$.

Our main result will now be presented.

Theorem 2.1. Let $T$ be a power-bounded operator on a Hilbert space $\mathcal{H}$. If $Q \in$ $\mathcal{L}(\mathcal{H})$ and $T Q=Q T$, then the following statements are equivalent:

(i) $\lim _{n \rightarrow \infty} \frac{1}{n}\left\|\sum_{k=0}^{n-1} \lambda^{-k} T^{k} Q\right\|=0$ for every $\lambda \in \sigma(T) \cap \mathbb{T}$,

(ii) $\lim _{n \rightarrow \infty}\left\|T^{n} Q\right\|=0$.

Moreover, if $Q=f(T)$ for some $f \in A^{+}(\mathbb{T})$, then (i) and (ii) are equivalent to

(iii) $f(\lambda)=0$ for every $\lambda \in \sigma(T) \cap \mathbb{T}$.

First we prove a lemma which leads us to the ergodic condition of the above theorem.

Lemma 2.2. Let $T$ be a power-bounded operator on a complex Banach space $\mathcal{X}$ and let $f \in A^{+}(\mathbb{T})$. Then, for every $\lambda \in \mathbb{T}$, we have

$$
\lim _{n \rightarrow \infty} \frac{1}{n}\left\|\sum_{k=0}^{n-1} \lambda^{-k} T^{k}(f(T)-f(\lambda) I)\right\|=0 .
$$

Proof. Taking the Taylor expansion $f(z)=\sum_{m=0}^{\infty} a_{m} z^{m}$ of $f$ and changing the order of summation, we have

$$
\begin{aligned}
\frac{1}{n} \sum_{k=0}^{n-1} \lambda^{-k} T^{k}(f(T)-f(\lambda) I) & =\frac{1}{n} \sum_{k=0}^{n-1} \lambda^{-k} T^{k}\left(\sum_{m=0}^{\infty} a_{m}\left(T^{m}-\lambda^{m} I\right)\right) \\
& =\sum_{m=0}^{\infty} a_{m} \lambda^{m}\left(\frac{1}{n} \sum_{k=0}^{n-1}\left(\lambda^{-(k+m)} T^{k+m}-\lambda^{-k} T^{k}\right)\right)
\end{aligned}
$$


Next, pick an $\varepsilon>0$. Let us choose an index $M \in \mathbb{N}$ such that $\sum_{m=M}^{\infty}\left|a_{m}\right| \leq \varepsilon$. Then we may infer that (with $\triangle$ denoting the symmetric difference of two sets)

$$
\begin{aligned}
\left\|\frac{1}{n} \sum_{k=0}^{n-1} \lambda^{-k} T^{k}(f(T)-f(\lambda) I)\right\| \leq & \sum_{m=0}^{M-1}\left|a_{m}\right|\left\|\frac{1}{n} \sum_{k=0}^{n-1}\left(\lambda^{-(k+m)} T^{k+m}-\lambda^{-k} T^{k}\right)\right\| \\
& +\sum_{m=M}^{\infty}\left|a_{m}\right|\left\|\frac{1}{n} \sum_{k=0}^{n-1}\left(\lambda^{-(k+m)} T^{k+m}-\lambda^{-k} T^{k}\right)\right\| \\
\leq & \|f\|_{1} L \max _{1 \leq m \leq M} \frac{\operatorname{card}(\{1, \ldots, n\} \triangle\{m+1, \ldots, m+n\})}{n} \\
& +2 L \varepsilon \\
\leq & \|f\|_{1} 2 L M n^{-1}+2 L \varepsilon<4 L \varepsilon
\end{aligned}
$$

provided $n$ is sufficiently large, where $\|f\|_{1}:=\sum_{m=0}^{\infty}\left|a_{m}\right|$ and $L:=\sup \left\{\left\|T^{k}\right\|: k=\right.$ $0,1,2, \ldots\}$. Since $\varepsilon$ was arbitrarily chosen, the lemma is proved.

The uniform ergodic theorem tells us that $\frac{1}{n} \sum_{k=0}^{n-1} T^{k}$ tends to zero in norm if and only if 1 is in the resolvent set of $T$ (cf. [16, Theorem 2.7]). With this result, the next corollary of the above lemma is straightforward.

Corollary 2.3. Let $T$ be a power-bounded operator on a Banach space $\mathcal{X}$ and $f \in A^{+}(\mathbb{T})$. Then, for each $\lambda \in \sigma(T) \cap \mathbb{T}$,

$$
f(\lambda)=0 \text { if and only if } \lim _{n \rightarrow \infty} \frac{1}{n}\left\|\sum_{k=0}^{n-1} \lambda^{-k} T^{k} f(T)\right\|=0 .
$$

In the following lemma we shall prove the theorem for an arbitrary isometry. Recall that Douglas's extension theorem ([7]) states that any isometry $V$ on a Banach space $\mathcal{X}$ can be extended to a surjective isometry on a larger space. This result had already been well known in the Hilbert space setting (cf. [12]). Later, C.J.K. Batty and S. Yeates gave a different construction in 2] which preserves the structure of the original space in most cases (for instance, when $\mathcal{X}$ is a Hilbert space or superreflexive). In addition, their construction makes it possible to define an extension of the commutant of $V$; they have shown that there exists a unital isometric algebra homomorphism from the commutant of $V$ into the commutant of its surjective extension [2, Proposition 3.5]. Then, under this homomorphism, the spectrum of an element of the commutant contains the spectrum of its image. (The reader should see [5] for a similar construction in the Hilbert space case.) Below we shall make use of these results.

Lemma 2.4. Let $V$ be an isometry on a Hilbert space $\mathcal{H}$. Suppose that $Q \in \mathcal{L}(\mathcal{H})$ and $Q V=V Q$. If

$$
\lim _{n \rightarrow \infty} \frac{1}{n}\left\|\sum_{k=0}^{n-1} \lambda^{-k} V^{k} Q\right\|=0
$$

holds for every $\lambda \in \sigma(V) \cap \mathbb{T}$, then $Q=0$.

Proof. Applying the extension theorem of Batty and Yeates, throughout the proof we can assume, without loss of generality, that $V$ is unitary.

It can be easily seen that $V Q^{*}=Q^{*} V$; hence the $C^{*}$-algebra $\mathcal{A}$ generated by $V$ and $Q Q^{*}$ is commutative. Let $\Sigma$ stand for the Gelfand spectrum of $\mathcal{A}$ and let 
$\Gamma_{\mathcal{A}}: \mathcal{A} \rightarrow C(\Sigma), A \mapsto \widehat{A}$ be the Gelfand transform of $\mathcal{A}$. Then the ergodic condition $\lim _{n \rightarrow \infty} \frac{1}{n}\left\|\sum_{k=0}^{n-1} \lambda^{-k} \widehat{V}^{k} \widehat{Q Q^{*}}\right\|_{\infty}=0$ is satisfied for every $\lambda$ in the range of $\widehat{V}$ because $\operatorname{ran} \widehat{V}=\sigma(V)$.

Let us assume that the nonnegative function $\widehat{Q Q^{*}} \in C(\Sigma)$ takes its maximum $q_{0}$ at $h_{0} \in \Sigma$. Choosing $\lambda_{0}:=\widehat{V}\left(h_{0}\right)$, we obtain for any $n \in \mathbb{N}$ that

$$
\frac{1}{n}\left\|\sum_{k=0}^{n-1} \lambda_{0}^{-k} \widehat{V}^{k} \widehat{Q Q^{*}}\right\|_{\infty} \geq \frac{1}{n}\left|\sum_{k=0}^{n-1} \lambda_{0}^{-k} \widehat{V}\left(h_{0}\right)^{k} \widehat{Q Q^{*}}\left(h_{0}\right)\right|=q_{0}
$$

This means that $q_{0}=0$ must hold, so $\widehat{Q Q^{*}}(h)=0$ for every $h \in \Sigma$. Hence $\sigma\left(Q Q^{*}\right)=$ $\{0\}$ and $Q Q^{*}=0$. This readily implies that $Q=0$.

Let $J$ be an infinite set and let $\mathcal{U}$ be a free ultrafilter on $J$. Now consider the Banach spaces $\ell^{\infty}(J, \mathcal{X})$ of all bounded functions from $J$ to $\mathcal{X}$, and $c_{0}(J, \mathcal{X} ; \mathcal{U})$ of all bounded functions from $J$ to $\mathcal{X}$ which converge to zero through the ultrafilter. The quotient space $\ell^{\infty}(J, \mathcal{X}) / c_{0}(J, \mathcal{X} ; \mathcal{U})$ is an ultrapower of the Banach space $\mathcal{X}$, which we will denote by $\mathcal{X}_{\mathcal{U}}$. Recall that if $\mathcal{X}$ is a Hilbert space, then $\mathcal{X}_{\mathcal{U}}$ is also a Hilbert space. In fact, the parallelogram identity is preserved under taking ultrapower, and the polarization identity tells us that the inner product on $\mathcal{X}_{\mathcal{U}}$ is given by

$$
\langle\bar{x}, \bar{y}\rangle_{\mathcal{U}}=\mathcal{U}-\lim _{n}\left\langle x_{n}, y_{n}\right\rangle
$$

where $\bar{x}, \bar{y}$ denote the equivalence classes of $\left\{x_{n}\right\}_{n}$ and $\left\{y_{n}\right\}_{n}$ in $\mathcal{X}_{\mathcal{U}}$.

For each $T \in \mathcal{L}(\mathcal{X})$, the ultrapower $T_{\mathcal{U}}$ of $T$ is defined by the formula

$$
T_{\mathcal{U}}\left(\left\{x_{n}\right\}+c_{0}(J, \mathcal{X} ; \mathcal{U})\right):=\left\{T x_{n}\right\}+c_{0}(J, \mathcal{X} ; \mathcal{U}) .
$$

Note that the mapping $T \mapsto T_{\mathcal{U}}$ is an isometric unital algebra homomorphism from $\mathcal{L}(\mathcal{X})$ into $\mathcal{L}\left(\mathcal{X}_{\mathcal{U}}\right)$ such that $\sigma(T)=\sigma\left(T_{\mathcal{U}}\right)$ (cf. [11, [19, Theorem V.1.4]).

Proof of Theorem 2.1. The implication (ii) $\Longrightarrow$ (i) is evident. To prove (i) $\Longrightarrow$ (ii), let us introduce a new semi-inner product on $\mathcal{H}$ by

$$
\langle x, y\rangle_{T}:=m\left(\left\{\left\langle T^{n} x, T^{n} y\right\rangle\right\}_{n}\right) \quad(x, y \in \mathcal{H}),
$$

where $m$ denotes a Banach limit. Forming the quotient space and its completion result in a Hilbert space $\mathcal{H}_{T}$, where $T$ acts as a $V$ isometry. If $X$ denotes the canonical embedding of $\mathcal{H}$ into $\mathcal{H}_{T}$, we obtain that $V X=X T$. In addition, the intertwining transformation $X$ naturally induces a contractive unital algebra homomorphism between the commutants of $T$ and $V$. That is, if $A$ is in $\{T\}^{\prime}$, the commutant of $T$, then there exists a unique operator $B \in\{V\}^{\prime}$ such that $X A=B X$ and $\|B\| \leq\|A\|$. It can also be shown that the mapping

$$
\gamma_{T}:\{T\}^{\prime} \rightarrow\{V\}^{\prime}, \quad A \mapsto B
$$

is a contractive unital algebra homomorphism and hence $\sigma(B) \subseteq \sigma(A)([14)$. Then (i) implies that $V$ and $\gamma_{T}(Q)$ satisfy the ergodic condition

$$
\lim _{n \rightarrow \infty} n^{-1}\left\|\sum_{k=0}^{n-1} \lambda^{-k} V^{k} \gamma_{T}(Q)\right\|=0
$$

for every $\lambda \in \sigma(V) \cap \mathbb{T}$. We can now apply Lemma 2.4 to this and then conclude that $\gamma_{T}(Q)=0$. Thus $m\left(\left\{\left\|Q T^{n} x\right\|^{2}\right\}_{n}\right)=m\left(\left\{\left\|T^{n} Q x\right\|^{2}\right\}_{n}\right)=0$ for every $x \in \mathcal{H}$, 
and hence $\inf _{n \geq 0}\left\|T^{n} Q x\right\|=0$ by the positivity of $m$. Therefore, the condition (i) in Theorem 2.1 implies that $\inf _{n \geq 0}\left\|T^{n} Q x\right\|=\lim _{n \rightarrow \infty}\left\|T^{n} Q x\right\|=0$ for every $x \in \mathcal{H}$.

Now let $\alpha:=\inf _{n \geq 0}\left\|T^{n} Q\right\|$ and $L:=\sup _{n \geq 0}\left\|T^{n}\right\|$. Choose a sequence of unit vectors $\left\{x_{k}\right\}_{k=0}^{\infty}$ in $\mathcal{H}$ such that $\left\|T^{k} Q x_{k}\right\| \geq \alpha / 2$ holds for every $k \in \mathbb{N}$. Then, of course, $\left\|T^{n} Q x_{k}\right\| \geq \alpha /(2 L)$ for every $n \leq k$. Taking a free ultrafilter $\mathcal{U}$ on $\mathbb{N}$, we can define the unit vector $\bar{x}=\left\{x_{k}\right\}_{k}+c_{0}(\mathbb{N}, \mathcal{H} ; \mathcal{U})$ in the ultrapower space $\mathcal{H}_{\mathcal{U}}$. Then, for any $n \in \mathbb{N}$, we have

$$
\begin{aligned}
\left\|T_{\mathcal{U}}^{n} Q \mathcal{U} \bar{x}\right\|=\left\|\left\{T^{n} Q x_{k}\right\}_{k}+c_{0}(\mathbb{N}, \mathcal{H} ; \mathcal{U})\right\| & =\mathcal{U}-\lim _{k}\left\|T^{n} Q x_{k}\right\| \\
& \geq \liminf _{k}\left\|T^{n} Q x_{k}\right\| \geq \frac{\alpha}{2 L} .
\end{aligned}
$$

However, applying the previous conclusion to $T_{\mathcal{U}}$ and $Q_{\mathcal{U}}$ which satisfy (i), we find that $T_{\mathcal{U}}^{n} Q_{\mathcal{U}}$ tends to zero strongly. This means that $\alpha$ must be zero; hence $\lim _{n \rightarrow \infty}\left\|T^{n} Q\right\|=0$ also holds, which is what we intended to show.

For $(\mathrm{i}) \Longleftrightarrow$ (iii), see Corollary 2.3 .

\section{ACKNowledgements}

I would like to thank Professor L. Kérchy for helpful comments and Professor L. Zsidó for several stimulating discussions.

\section{REFERENCES}

1. C.J.K. Batty, Asymptotic behaviour of semigroups of operators, in: Functional analysis and operator theory (J. Zemánek, ed.), Banach Center Publ., 30, Polish Acad. Sci., Warsaw, 1994, 35-52. MR.1285599 (95g:47058)

2. C.J.K. Batty and S. Yeates, Extensions of semigroups of operators, J. Operator Theory, 46 (2001), 139-157. MR.1862183 (2002j:47067)

3. C.J.K. Batty and Q.P. Vũ, Stability of strongly continuous representations of abelian semigroups, Math. Z., 209 (1992), 75-88. MR.1143215 (93d:47073)

4. H. Bercovici, On the iterates of a completely nonunitary contraction, Topics in operator theory: Ernst D. Hellinger memorial volume, Operator Theory Adv. Appl., 48, Birkhäuser, Basel, 1990, 185-188. MR.1207397 (94g:47019)

5. H. Bercovici, Commuting power bounded operators, Acta Sci. Math. (Szeged), 57 (1993), 55-64. MR 1243268 (94j:47008)

6. R. Chill and Y. Tomilov, Stability of operator semigroups: ideas and results, Banach Center Publ., 75, Polish Acad. Sci., Warsaw, 2007, 71-109. MR2336713 (2008m:47054)

7. R.G. Douglas, On extending commutative semigroups of isometries, Bull. London Math. Soc., 1 (1969), 157-159. MR0246153(39:7458)

8. J. Esterle, Quasimultipliers, representation of $H^{\infty}$, and the closed ideal problem for commutative Banach algebras, in: Radical Banach algebras and automatic continuity, Lecture Notes in Math., 975, Springer, Berlin, 1983, 66-162. MR697579 (85g:46067)

9. J. Esterle, E. Strouse and F. Zouakia, Theorems of Katznelson-Tzafriri type for contractions, J. Funct. Anal., 94 (1990), 273-287. MR.1081645 (92c:47016)

10. J. Esterle, E. Strouse and F. Zouakia, Stabilité asymptotique de certains semi-groupes d'opérateurs et idéaux primaires de $L^{1}\left(\mathbb{R}^{+}\right)$, J. Operator Theory, 28 (1992), 203-227. MR.1273043 (95f:43001)

11. S. Heinrich, Ultraproducts in Banach space theory, J. Reine Angew. Math., 313 (1980), 72-104. MR.552464 (82b:46013)

12. T. Ito, On the commutative family of subnormal operators, J. Fac. Sci. Hokkaido Univ. Ser. I, 14 (1958), 1-15. MR0107177(21:5902)

13. Y. Katznelson and L. Tzafriri, On power bounded operators, J. Funct. Anal., 68 (1986), 313-328. MR859138(88e:47006)

14. L. Kérchy, Isometric asymptotes of power bounded operators, Indiana Univ. Math. J., 38 (1989), 173-188. MR982576 (90f:47050) 
15. L. Kérchy and J. van Neerven, Polynomially bounded operators whose spectrum on the unit circle has measure zero, Acta Sci. Math. (Szeged), 63 (1997), 551-562. MR 1480498 (99a:47007)

16. U. Krengel, Ergodic theorems, de Gruyter, Berlin, 1985. MR.797411 (87i:28001)

17. H.S. Mustafayev, The Banach algebra generated by a contraction, Proc. Amer. Math. Soc., 134 (2006), 2677-2683. MR.2213747 (2006m:47013)

18. H.S. Mustafayev, The behavior of the radical of the algebras generated by a semigroup of operators on Hilbert space, J. Operator Theory, 57 (2007), 19-34. MR2301935 (2008b:47071)

19. H. H. Schaefer, Banach lattices and positive operators, Grundl. Math. Wiss., 215, SpringerVerlag, New York- Heidelberg-Berlin, 1974. MR0423039 (54:11023)

20. Q. P. Vũ, A short proof of the Y. Katznelson's and L. Tzafriri's theorem, Proc. Amer. Math. Soc., 115 (1992), 1023-1024. MR.1087468 (92j:47012)

21. Q. P. Vũ, Theorems of Katznelson-Tzafriri type for semigroups of operators, J. Funct. Anal., 103 (1992), 74-84. MR 1144683 (93e:47050)

22. J. Zemánek, On the Gelfand-Hille theorems, in: Functional analysis and operator theory (J. Zemánek, ed.), Banach Center Publ., 30, Polish Acad. Sci., Warsaw, 1994, 369-385. MR 1285622 (95f:47009)

Bolyai Institute, University of Szeged, Aradi vértanúk tere 1, H-6720 Szeged, HunGARY

E-mail address: lzoli@math.u-szeged.hu 